\title{
Decline of emergency admissions for cardiovascular and cerebrovascular events after the outbreak of COVID-19
}

\author{
Viktoria Schwarz ${ }^{1,2} \cdot$ Felix Mahfoud $^{2} \cdot$ Lucas Lauder $^{2} \cdot$ Wolfgang Reith $^{3} \cdot$ Stefanie Behnke ${ }^{5}$ Sigrun Smola ${ }^{4}$. \\ Jürgen Rissland ${ }^{4} \cdot$ Thorsten Pfuhl $^{4} \cdot$ Bruno Scheller $^{2} \cdot$ Michael Böhm $^{2} \cdot$ Sebastian Ewen $^{1,2}$ (])
}

Received: 14 May 2020 / Accepted: 7 June 2020 / Published online: 4 August 2020

(c) The Author(s) 2020

\begin{abstract}
Background The spread of the novel coronavirus SARS-CoV-2 and the guidance from authorities for social distancing and media reporting lead to significant uncertainty in Germany. Concerns have been expressed regarding the underdiagnosing of harmful diseases. We explored the rates of emergency presentations for acute coronary syndrome (ACS) and acute cerebrovascular events (ACVE) before and after spread of SARS-CoV-2.

Methods We analyzed all-cause visits at a tertiary university emergency department and admissions for ACS and ACVE before (calendar weeks 1-9, 2020) and after (calendar weeks 10-16, 2020) the first coronavirus disease (COVID-19) case in the region of the Saarland, Germany. The data were compared with the same period of the previous year.

Results In 2020 an average of 346 patients per week presented at the emergency department whereas in 2019 an average of 400 patients presented up to calendar week 16 ( $p=0.018$; whole year $2019=395$ patients per week). After the first COVID19 diagnosis in the region, emergency department visit volume decreased by 30\% compared with the same period in 2019 $(p=0.0012)$. Admissions due to ACS decreased by $41 \%$ ( $p=0.0023$ for all; $\Delta-71 \%(p=0.007)$ for unstable angina, $\Delta$ $-25 \%(p=0.42)$ for myocardial infarction with ST-elevation and $\Delta-17 \%(p=0.28)$ without ST-elevation) compared with the same period in 2019 and decreased from 142 patients in calendar weeks 1-9 to 62 patients in calendar weeks 10-16. ACVE decreased numerically by $20 \%$ [ $p=0.25$ for all; transient ischemic attack: $\Delta-32 \%(p=0.18)$, ischemic stroke: $\Delta$ $-23 \%(p=0.48)$, intracerebral haemorrhage: $\Delta+57 \%(p=0.4)$ ]. There was no significant change in ACVE per week $(p=0.7)$ comparing calendar weeks 1-9 (213 patients) and weeks 10-16 (147 patients). Testing of 3756 samples was performed to detect 58 SARS-CoV-2 positive patients (prevalence $1,54 \%$, thereof one patient with myocardial and two with cerebral ischemia) up to calendar week 16 in 2020.

Conclusions The COVID-19 pandemic was associated with a significant decrease in all-cause admission and admissions due to cardiovascular events in the emergency department. Regarding acute cerebrovascular events there was a numerical decrease but no significant difference.
\end{abstract}

Keywords COVID-19 $\cdot$ SARS-CoV-2 Acute coronary syndrome $\cdot$ Cardiovascular events $\cdot$ Cerebrovascular events

Viktoria Schwarz

viktoria.schwarz@uks.eu

Sebastian Ewen

Sebastian.Ewen@uks.eu

1 Emergency Department, Saarland University Medical Center, Homburg, Germany

2 Clinic of Internal Medicine III (Cardiology, Angiology and Intensive Care Medicine), Saarland University Medical Center, Universitätsklinikum des Saarlandes, Saarland University, Kirrberger Str. 100, Geb. 41, 66421 Homburg, Saar, Germany
3 Department of Neuroradiology, Saarland University Medical Center, Homburg, Germany

4 Institute of Virology, Saarland University Medical Center, Homburg, Germany

5 Department of Neurology, Saarland University Medical Center, Homburg, Germany 


\section{Introduction}

The ongoing pandemic of the novel coronavirus SARSCoV-2 disease (COVID-19) unsettles people worldwide and has led to 287,399 deaths globally by May 13, 2020 [1]. To protect the local health system from overload and to maintain intensive care capacities for COVID-19 patients, elective admissions have been reduced and postponed. In addition to hygiene advices, the World Health Organization (WHO) strongly recommends limiting face-to-face contact with others via social distancing [2]. Subsequently, changes in the pattern of hospital admissions have been observed [3-6]. Valuable time passes with the ubiquitous differential diagnosis of respiratory insufficiency caused by COVID-19, which is frequently aimed to exclude before detailed examinations (e.g., physical examination, ultrasound, computed tomography, coronary angiography) take place [7]. Therefore, concerns have been raised regarding underdiagnosing of harmful but treatable cardiovascular diseases.

\section{Methods}

\section{Study design and patients}

We analyzed ICD-10-GM (International Statistical Classification of Diseases and Related Health Problems, 10th Revision, German Modification) codes from discharge letters at Saarland University Medical Center, Germany, during calendar weeks 1-16 of the years 2019 and 2020, respectively. We assessed the all-cause visit volume at the emergency department of Internal Medicine (aged $\geq 18$ years) and Surgery (aged $\geq 0$ years). Digital records of patients admitted for acute coronary syndrome (ACS), including unstable angina pectoris as well as myocardial infarction with (STEMI) or without (NSTEMI) ST-elevation were evaluated. Similarly, the number of acute cerebrovascular events (ACVE), including transient ischemic attack, ischemic stroke, and intracerebral haemorrhage were evaluated. We compared calendar weeks 1-9 before the first COVID-19 case in the region of Saarland (1st case on March 3rd, calendar week 10) with calendar weeks 10-16, during which the infection numbers in the region increased. Focus was also given on the timing of the shutdown in the Saarland (March 16th, calendar week 12). To reduce bias due to seasonal effects, we compared the visit volume with the same period of the previous year.

\section{RT-PCR-based diagnostic of COVID-19}

The indication for COVID-19 testing was performed according to the recommendations of the Robert Koch Institute
[8]. COVID-19 confirmation test was performed by isolated RNA from eSwabs (Copan Italia, Brescia, Italy) using the NucliSens easy MAG Instrument (bioMeriéux Deutschland, Nürtingen, Germany) following the manufacturers' instructions. PCR amplification used the RealStar SARS-CoV-2 RT-PCR Kit 1.0 RUO (Altona Diagnostics, Hamburg, Germany) on a Light Cycler 480 II Real-Time PCR Instrument (Roche Diagnostics Deutschland, Mannheim, Germany) according to the manufacturers' instructions [9]. From week 12 on COVID-19 confirmation was additionally performed with the cobas ${ }^{\circledR}$ SARS-CoV-2 Test for the cobas ${ }^{\circledR}$ 6800 instrument (Roche Diagnostics GmbH, Mannheim, Germany) according to manufacturers' instructions. Only tests from patients at the Saarland University Medical Center were included in the analyses, tests from staff were excluded.

\section{Statistics}

Data are presented as mean unless otherwise specified. Categorical variables are presented as numbers (\%). Variables were tested for normality utilizing the Shapiro-Wilk test. For continuous variables, between-group comparisons were performed using the Mann-Whitney or Wilcoxon rank-sum test and Kruskal-Wallis-test where appropriate. Significance tests were two-tailed with $p<0.05$ considered significant. All statistical analyses were performed using GraphPad Prism version 8.2.1 (GraphPad Software, La Jolla, CA, USA).

\section{Results}

\section{Emergency department visit volume}

At the emergency department in 2020 an average of 346 patients per week presented up to calendar week 16 whereas in 2019 an average of 400 patients per week presented ( $p=0.018$ ) over the same period of time. In 2020, admissions at the emergency department decreased by calendar week 8 parallel to the occasional detection of COVID-19 in Germany (Fig. 1). After verification of the first patient with COVID-19 in the Saarland region on March 3, 2020 (calendar week 10), the number of admissions dropped, regardless of the reason for admission (average of 397 patients per week in calendar weeks 1-9 vs. average of 279 patients per week in calendar weeks 10-16, $p=0.016$ ) (Fig. 2a).There was no significant difference for the average visit volume when comparing weeks $1-9$ of 2020 with 2019. After the first COVID-19 diagnosis in the region, all-cause emergency department visit volume decreased by $30 \%$ compared with the same period in 2019 (1954 patients in 2020 vs. 2773 patients in 2019 at calendar weeks $10-16, p=0.0012$ ) and tended to further decrease by $36 \%$ after the shutdown in 


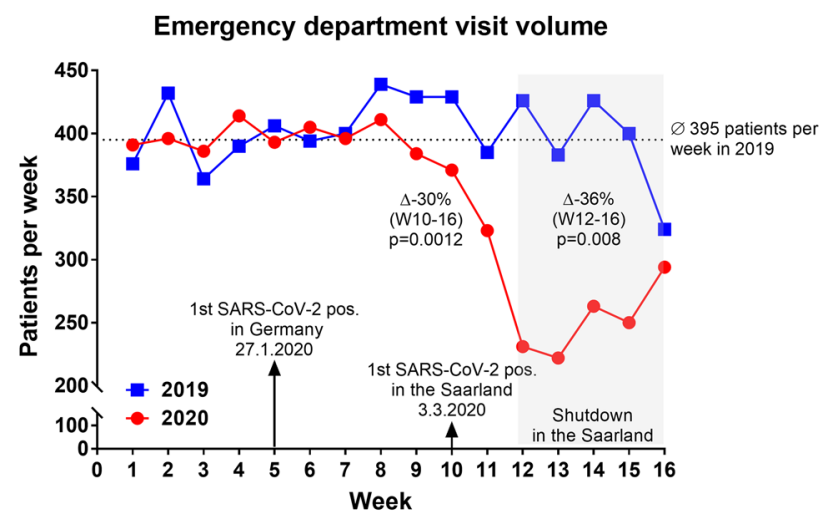

Fig. 1 Emergency department visit volume. Comparison of the number of patients during calendar weeks $1-16$ in 2019 and 2020

the Saarland compared with the same period in 2019 (1260 patients in 2020 vs. 1959 patients in 2019 at calendar weeks $12-16, p=0.008)$. In parallel to the regional shutdown, the nadir was reached at calendar weeks $12-13$. Following calendar week 14, the visit volume at the emergency department increased again but remained significantly lower compared with the previous year.

\section{Acute coronary syndrome in the emergency department}

After the first laboratory-confirmed SARS-CoV-2 infection in calendar week 10 of 2020 in the Saarland, the number of patients diagnosed with an ACS decreased significantly by $41 \%$ compared with the previous year (62 patients in $2020 \mathrm{vs}$. 105 patients in 2019 at calendar weeks $10-16, p=0.0023$ ) (Fig. 3). During the shutdown in calendar weeks 12-16, there was a further reduction of patients who admitted with ACS compared with the previous year $(\Delta-48 \%, 39$ patients in 2020 vs. 75 patients in 2019 at calendar weeks 12-16, $p=0.008$ ). There was no significant difference in admission rates when comparing week 1-9 of the years 2020 to
2019. Regarding the different entities of ACS, we observed a significant decrease in unstable angina by $71 \%$ after the first confirmed COVID-19 patient in the region (12 patients in $2020 v s .42$ patients in $2019, p=0.007)$ respectively a decrease by $85 \%$ during the lockdown (4 patients in $2020 \mathrm{vs}$. 27 patients in $2019, p=0.008)$ compared with the previous year. The decline in STEMI $(\Delta-25 \%$ in calendar weeks $10-16,21$ patients in 2020 vs. 28 patients in 2019, $p=0,42$; $\Delta-41 \%$ in calendar weeks $12-16,13$ patients in $2020 v s$. 22 patients in $2019, p=0.25)$ and NSTEMI $(\Delta-17 \%$ in calendar weeks $10-16,29$ patients in 2020 vs. 35 patients in $2019, p=0.28 ; \Delta-19 \%$ in calendar weeks $12-16,22$ patients in 2020 vs. 29 patients in 2019, $p=0.4$ ) was not significant. However, in weeks 1-9 more ACS were treated compared with period after the first confirmed COVID-19 case in the region (142 patients in calendar weeks 1-9 vs. 62 patients in calendar weeks $10-16, p=0.0007$ ) (Fig. 2b).

\section{Acute cerebrovascular events}

Admissions due to ACVE, including transient ischemic attack, ischemic stroke, and intracerebral haemorrhage were numerically reduced by $20 \%$ at calendar week 10 (147 patients in 2020 vs. 183 patients in $2019, p=0.25$ ) and by $31 \%$ during shutdown (98 patients in $2020 \mathrm{vs}$. 143 patients in 2019, $p=0.14$ ) compared with the same period last year (Fig. 4). Looking at the individual entities at calendar week 10-16 and under lockdown conditions compared to the same period in 2019 there was a non-significant reduction by $32 \%$ (41 patients in 2020 $v s .60$ patients in 2019, $p=0.18$ ) and $34 \%$ (31 patients in 2020 vs. 47 patients in 2019, $p=0.2$ ) of transient ischemic attack, a 23\% (84 patients in $2020 \mathrm{vs.} 109$ patients in $2019, p=0.48$ ) and $34 \%$ (57 patients in $2020 v s .86$ patients in 2019, $p=0.25$ ) non-significant reduction of ischemic strokes as well as a $57 \%$ (22 patients in $2020 \mathrm{vs}$. 14 patients in $2019, p=0.4)$ and $0 \%$ (10 patients in 2020 vs. 10 patients in $2019, p=1$ ) also not significant increase
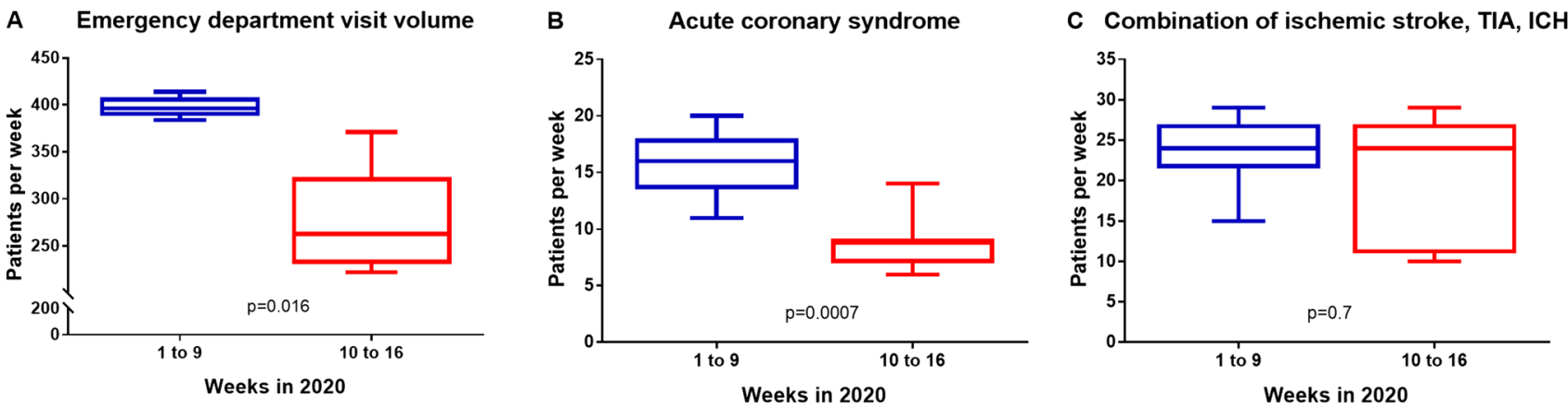

Fig. 2 Box plot of patients at calendar weeks 1-9 and 10-16 in the year 2020. a Emergency department visit volume. b Acute coronary syndrome. c Combination of ischemic stroke, transient ischemic attack (TIA) and intracerebral haemorrhage (ICH) 

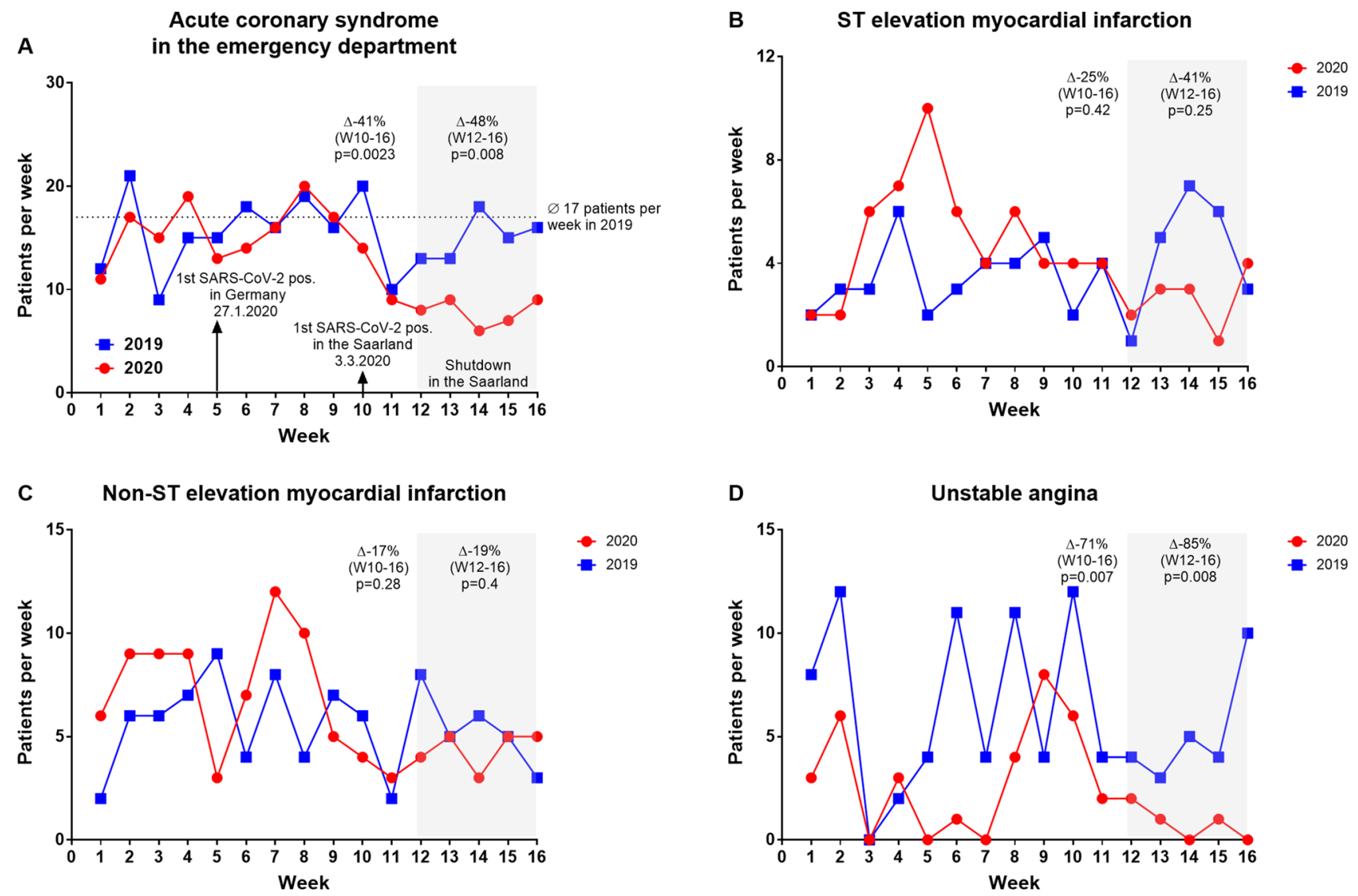

Fig. 3 Acute coronary syndrome. a Accumulated number of patients with acute coronary syndrome in the emergency department at calendar weeks 1-16 in the year 2019 and 2020. Number of patients

in intracerebral haemorrhage. There was no significant difference when comparing the mean number of presentations per week for acute cerebral events at week 1-9 and $10-16$ of the year 2020 (24 patients per week in $2020 \mathrm{vs}$. 8 patients per week in 2019, $p=0.7$ ) (Fig. 2c).

\section{Virological test volume for COVID-19}

With spread of the infection the test volume for SARSCoV-2 testing increased week to week (one test in calendar week 6, 965 tests in calendar week 16; Fig. 5). In our analyses during end of week 16 in 2020, testing of 3765 samples detected 58 positive patients. The overall rate of positive tests from patients of the Saarland University Medical Center up to calendar week 16 was $1.54 \%$. We identified one patient with acute myocardial infarction and one patient with ACVE with coincidental SARS-CoV-2 infection. One patient with previously known COVID-19 infection was admitted with an acute cerebrovascular event.

at calendar weeks $1-16$ in the year 2019 and 2020 with myocardial infarction with (b, STEMI) and without (c, NSTEMI) ST-elevation and unstable angina $(\mathbf{d})$

\section{Discussion}

At the Saarland University hospital, a tertiary referral center, a significant decrease in medical contacts at the emergency department and the number of patients presenting with ACS was documented during the first wave of the COVID-19 pandemic. This was mainly driven due to an $85 \%$ decrease in admission for unstable angina during shutdown conditions whereas the reduction in admissions caused by myocardial infarction was not significant. Regarding acute cerebrovascular events there was a numerically reduction in our analyses.

During the past weeks, reports on increasing numbers of COVID-19 positive patients in China, US and Europe dominated the reporting in the lay press and resulted in pressure on health care systems like in Northern Italy. This coverage led to great fear and uncertainty in several countries, including Germany [10]. In England attendances at the emergency department have fallen by $25 \%$ the week after the prime minister Boris Johnson announced a lockdown on 23 March [11]. Similar decline of emergency department visits could 

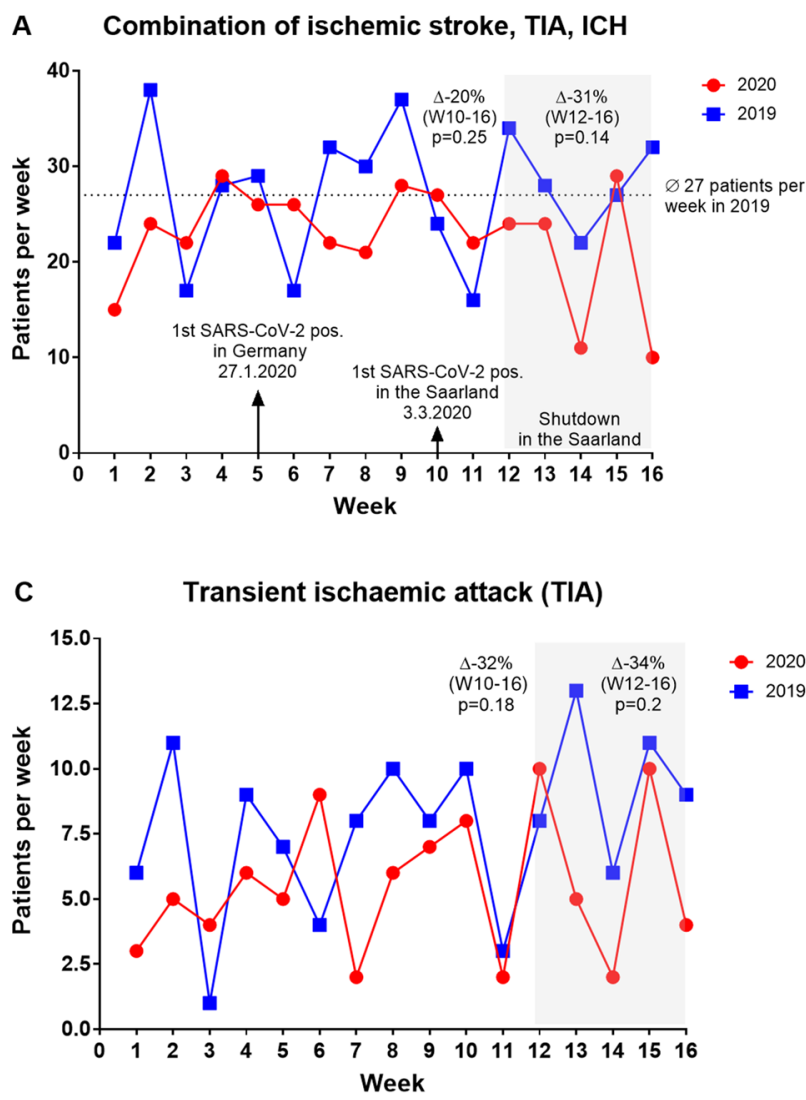

Fig. 4 Acute cerebrovascular events. a Accumulated number of patients with ischemic stroke, transient ischemic attack (TIA) and intracerebral haemorrhage (ICH) at calendar weeks 1-16 in the year
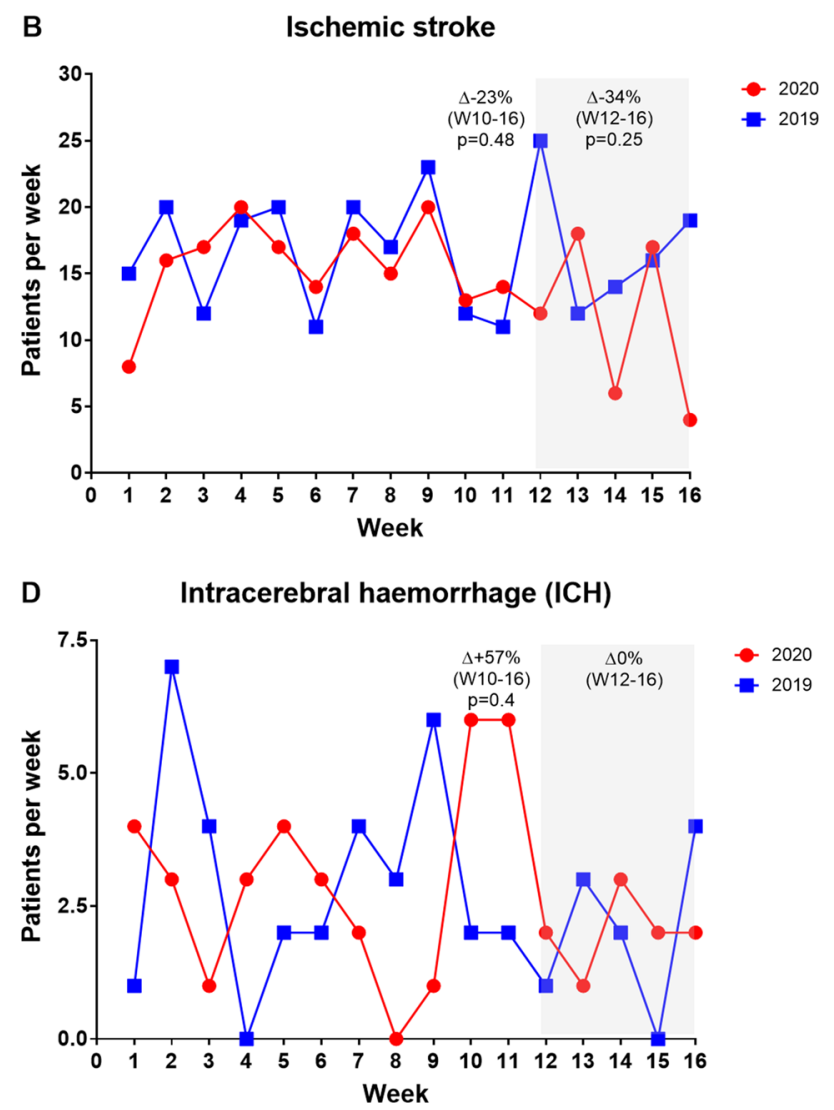

2019 and 2020. Number of patients at calendar weeks 1-16 in the year 2019 and 2020 with ischemic stroke (b), TIA (c) and ICH (d)

also be observed for paediatric emergency department visits [6]. To exclude a seasonal effect in the analyses, we compared the visit volume with the same period of the previous year. Proportionally more patients who had been admitted to the emergency department subsequently required inpatient treatment, indicating that consultations for less severe reasons decreased. In calendar weeks 15-16, the emergency department visit volume graphs of 2019 and 2020 get closer, which may have several reasons like behavior and travelling attitudes. Exemplarily, Christmas and Midsummer holidays have been associated with higher risk of myocardial infarction; a weekend-effect with delay in coronary angiography for NSTEMI resulting in a higher mortality has been described $[12,13]$. Behavior during the Easter holidays may have been different in 2020 due to the government restrictions in Germany. In 2019 (Easter at calendar week 16), the festivity with the family and/or a short vacation was obvious,

Fig. 5 Virological test volume for SARS-CoV-2 for patients at the Saarland University Medical Center 
in the family itself and a relevant number of people were located abroad. In 2020 (Easter at calendar week 15) neither a family visit nor a journey was allowed due to the lockdown in our region. Patients who had previously postponed their doctor's appointment may have gone to see a doctor due to the increasing level of suffering.

We found an "atypical" low number of cardiovascular events compared with the weeks before the regional outbreak of the pandemic and compared with the same period of the previous year. A similar trend was observed in cardiovascular centers in Austria [14], the severely affected Northern Italy [3] and Iran [15]. It can be assumed by a stable incidence of patients having an ACS that patients abstain from seeing a doctor despite symptoms of acute myocardial ischemia. This is in line with the increasing number of outof-hospital cardiac arrest in Italy compared with the same period in 2019 [4]. In COVID-19 patients, cardiac injury is a common feature whereby the mechanisms have not yet been finally clarified [16]. In our analyses, there was only one patient with myocardial infarction who was tested positive for the virus, but regarding a total number of 62 patients presenting with ACS the ratio was 1,6\%-and is therefore in the area of the overall prevalence in our hospital. A significant reduction in catheterization laboratory activations during COVID-19 pandemic due to a decline in patients with STEMI was observed in the US [17].

Although the difference was not statistically significant, we documented a numerically lower number of transient ischemic attacks and ischemic strokes. According to the World Stroke Organization there is a significant fall in stroke presentations globally [5]. One may speculate that patients with mild or moderate symptoms refrain from consulting health care providers. The increase in intracerebral haemorrhage could be (1) directly attributed to underdiagnosed and therefore undertreated cerebral ischemia, (2) less strictly controlled blood pressure values due to reduced contacts with doctors or (3) an increase of accidents in the household (e.g., due to self-made construction work or family violence during the shutdown and government restrictions) [18-21].

Coagulopathy, inflammatory responses, and endothelial dysfunction are associated with COVID-19 [22-24]. As a result of this, with (symptomatic as well as asymptomatic) spread of SARS-CoV-2 infection an increased rate of acute cardiovascular and acute cerebrovascular events can be assumed [25]. The reduction in ED numbers was not substantiated by an objective increase of COVID-19 infections. Despite testing the day of admission, by now, we identified only one patient with acute myocardial infarction and one patient with acute cerebrovascular event with coincidental SARS-CoV-2 infection. In addition, only one patient with previously known COVID-19 was admitted with an acute cerebrovascular event. Given the overall low number of positive test at our hospital, patients' concern about an increased risk of infection in the hospital appears unsubstantiated.

As a result of the legal provisions that no non-urgent or preventive medical examinations should take place during the shutdown, it can be assumed that a high rate of underdiagnosed diseases can be expected. The general recommendation to reduce social contacts in everyday life wherever and whenever possible led more people to stay at their presumed safe homes. This recommendation could have impaired respectively delayed scheduled and unscheduled doctor visits. Whether the rate of successfully treated COVID-19-positive patients outweighs the high number of undiagnosed diseases caused by the shutdown remains to be answered. It would be fatal if there would be an increase in deaths-especially in those without COVID-19 having harmful, but treatable diseases.

\section{Limitations}

Patients presented directly at the department of neurology and pediatrics or transferred directly to specialized departments were not included in the analysis of the total number of patients presented at the central emergency department at the Saarland University Medical Center.

\section{Conclusions}

The COVID-19 pandemic is associated with a significant decrease in all-cause admission and admissions due to acute cardiovascular and potentially cerebrovascular events in the emergency department. Regarding acute cerebrovascular events there was a numerical difference which needs to be scrutinized in large sample series. Increase of test capacity adapted to the pandemic process up to the general testing for inpatients has been succeeded in order to be able to maintain standard medical care and offers chances to restart indicated preventive medicine. Essential part of successful medical treatment maintains the step that the patient comes to see a doctor at all. As a result of these observations, regional media campaigns as already started are important to encourage patients to continue to see a doctor if they have healthrelated problems.

Acknowledgments Open Access funding provided by Projekt DEAL.

Funding None.

Data availability All data is available and can be provided if requested. 


\section{Compliance with ethical standards}

Conflicts of interests VS, LL, WR, SB, SS, JR, TP, BS and SE have nothing to declare in relation to the present work. FM and MB are supported by Deutsche Gesellschaft für Kardiologie (DGK), and Deutsche Forschungsgemeinschaft (SFB TRR219).

Ethics approval/Consent to participate/Consent for publication The study was conducted in accordance with the Declaration of Helsinki, and local ethics committees approved the study protocol. The provided information is anonymous and does not expose the identity of the patient. Due to the retrospective nature of the study all the procedures being performed were part of the routine care.

Open Access This article is licensed under a Creative Commons Attribution 4.0 International License, which permits use, sharing, adaptation, distribution and reproduction in any medium or format, as long as you give appropriate credit to the original author(s) and the source, provide a link to the Creative Commons licence, and indicate if changes were made. The images or other third party material in this article are included in the article's Creative Commons licence, unless indicated otherwise in a credit line to the material. If material is not included in the article's Creative Commons licence and your intended use is not permitted by statutory regulation or exceeds the permitted use, you will need to obtain permission directly from the copyright holder. To view a copy of this licence, visit http://creativecommons.org/licenses/by/4.0/.

\section{References}

1. World health organization. Coronavirus disease 2019 (COVID19) situation report: 114. 2020. https://www.who.int/docs/defau 1t-source/coronaviruse/situation-reports/20200501-covid-19-sitre p.pdf?sfvrsn=742f4a18_4. Accessed 13 May 2020

2. World health organization. Coronavirus disease (COVID-19) advice for the public. 2020. https://www.who.int/emergencies/diseases/ novel-coronavirus-2019/advice-for-public. Accessed 13 May 2020

3. De Filippo O, D’Ascenzo F, Angelini F, Bocchino PP, Conrotto F, Saglietto A, Secco GG, Campo G et al (2020) Reduced rate of hospital admissions for ACS during Covid-19 outbreak in Northern Italy. N Engl J Med. https://doi.org/10.1056/NEJMc2009166 (published online)

4. Baldi E, Sechi GM, Mare C, Canevari F, Brancaglione A, Primi R, Klersy C, Palo A et al (2020) Out-of-hospital cardiac arrest during the Covid-19 outbreak in Italy. N Engl J Med. https://doi. org/10.1056/NEJMc2010418 (published online)

5. Markus HS, Brainin M (2020) COVID-19 and stroke-A global World Stroke Organization perspective. Int J Stroke. https ://doi.org/10.1177/1747493020923472 (published online, 1747493020923472)

6. Lazzerini M, Barbi E, Apicella A, Marchetti F, Cardinale F, Trobia G (2020) Delayed access or provision of care in Italy resulting from fear of COVID-19. Lancet Child Adolesc Health 4(5):e10e11. https://doi.org/10.1016/s2352-4642(20)30108-5

7. Custodis F, Schwarzkopf K, Weimann R, Spüntrup E, Böhm M, Laufs U (2020) A SARS-Cov2-negative corona victim. Clin Res Cardiol. https://doi.org/10.1007/s00392-020-01668-z

8. Robert Koch Institut. COVID-19 (Coronavirus SARS-CoV-2), 2020. https://www.rki.de/DE/Content/InfAZ/N/Neuartiges_Coron avirus $/ \mathrm{nCoV}$.html. Accessed 10 May 2020

9. Lohse S, Pfuhl T, Berkó-Göttel B, Rissland J, Geißler T, Gärtner B, Becker SL, Schneitler S et al (2020) Pooling of samples for testing for SARS-CoV-2 inasymptomatic people. Lancet Infect Dis. https://doi.org/10.1016/S1473-3099(20)30362-5
10. Krumholz HM (2020) Where have all the heart attacks gone? The New York Times https://www.nytimes.com/2020/04/06/well/live/ coronavirus-doctors-hospitals-emergency-care-heart-attack-strok e.html. Accessed 1 May 2020

11. Thornton $\mathbf{J}$ (2020) Covid-19: A and E visits in England fall by $25 \%$ in week after lockdown. BMJ 369:m1401. https://doi.org/10.1136/ bmj.m1401

12. Mohammad MA, Karlsson S, Haddad J, Cederberg B, Jernberg T, Lindahl B, Fröbert O, Koul S et al (2018) Christmas, national holidays, sport events, and time factors as triggers of acute myocardial infarction: SWEDEHEART observational study 1998-2013. BMJ 363:k4811. https://doi.org/10.1136/bmj.k4811

13. Eindhoven DC, Wu HW, Kremer SWF, van Erkelens JA, Cannegieter SC, Schalij MJ, Borleffs CJW (2018) Mortality differences in acute myocardial infarction patients in the Netherlands: the weekend-effect. Am Heart J 205:70-76. https://doi.org/10.1016/j.ahj.2018.07.015

14. Metzler B, Siostrzonek P, Binder RK, Bauer A, Reinstadler SJ (2020) Decline of acute coronary syndrome admissions in Austria since the outbreak of COVID-19: the pandemic response causes cardiac collateral damage. Eur Heart J. https://doi.org/10.1093/ eurheartj/ehaa314

15. Abdi S, Salarifar M, Mortazavi SH, Sadeghipour P, Geraiely B (2020) COVID-19 sends STEMI to quarantine!? Clin Res Cardiol. https://doi.org/10.1007/s00392-020-01664-3 (published online)

16. Böhm M Frey N Giannitsis E Sliwa K Zeiher A (2020) Coronavirus disease 2019 (COVID-19) and its implications for cardiovascular care: expert document from the German Cardiac Society and the World Heart Federation. Clin Res Cardiol (published online)

17. Garcia S, Albaghdadi MS, Meraj PM, Schmidt C, Garberich R, Jaffer FA, Dixon S, Rade JJ et al (2020) Reduction in ST-segment elevation cardiac catheterization laboratory activations in the United States during COVID-19 pandemic. J Am Coll Cardiol 75(22):2871-2872. https://doi.org/10.1016/j.jacc.2020.04.011

18. Hornig CR, Dorndorf W, Agnoli AL (1986) Hemorrhagic cerebral infarction-a prospective study. Stroke 17(2):179-185. https://doi. org/10.1161/01.str.17.2.179

19. Campbell AM (2020) An increasing risk of family violence during the Covid-19 pandemic: strengthening community collaborations to save lives. Forensic Sci Int Rep 2:100089. https://doi. org/10.1016/j.fsir.2020.100089

20. Sakaniwa R, Tromp J, Shirai K, Yamagishi K, Tamakoshi A, Iso H (2020) The association of conventionally medicated systolic and diastolic blood pressure level and mortality from cardiovascular disease: is the lower the better in high stroke population? Clin Res Cardiol. https://doi.org/10.1007/s00392-019-01587-8 (published online)

21. Meredith PA (1996) Therapeutic implications of drug "holidays'. Eur Heart J. https://doi.org/10.1093/eurheartj/17.suppl_a.21 (17 Suppl A)

22. Klok FA, Kruip M, van der Meer NJM, Arbous MS, Gommers D, Kant KM, Kaptein FHJ, van Paassen J et al (2020) Incidence of thrombotic complications in critically ill ICU patients with COVID-19. Thromb Res. https://doi.org/10.1016/j.throm res.2020.04.013 (published online)

23. Varga Z, Flammer AJ, Steiger P, Haberecker M, Andermatt R, Zinkernagel AS, Mehra MR, Schuepbach RA et al (2020) Endothelial cell infection and endotheliitis in COVID-19. Lancet 395(10234):1417-1418. https://doi.org/10.1016/s0140 -6736(20)30937-5 (published online)

24. Guzik TJ, Mohiddin SA, Dimarco A, Patel V, Savvatis K, MarelliBerg FM, Madhur MS, Tomaszewski M et al (2020) COVID-19 and the cardiovascular system: implications for risk assessment, diagnosis, and treatment options. Cardiovasc Res. https://doi. org/10.1093/cvr/cvaa106 (published online)

25. Hinterseer M, Zens M, Wimmer RJ, Delladio S, Lederle S, Kupatt C, Hartmann B (2020) Acute myocardial infarction due to coronary stent thrombosis in a symptomatic COVID-19 patient. Clin Res Cardiol. https://doi.org/10.1007/s00392-020-01663-4 